March 4, 1998

\title{
Charge Determination of High Energy Electrons and Nuclei by Synchrotron Radiation with AMS
}

\author{
H. Hofer and M. Pohl \\ ETH Zürich
}

\begin{abstract}
We investigate the possibilities to identify the charge of $\mathrm{TeV}$ electrons and $\mathrm{PeV}$ nuclei using their synchrotron radiation in the earth's magnetic field. Characteristics of synchrotron radiation photons are evaluated and methods of detection are discussed.
\end{abstract}

To be published in Nucl. Inst. Meth. 


\section{Introduction}

Cosmic electrons in the $\mathrm{TeV}$ energy range are generally assumed to come from point sources in our galaxy. At high energies between 0.1 and $1 \mathrm{TeV}$, only a few nearby sources saturate the electron spectrum [1]. Above $1 \mathrm{TeV}$, there are no measurements. Several hounded electrons per year are expected above this energy and inside the acceptance of the Alpha Magnetic Spectrometer, AMS [2].

Most primary positrons would, on the other hand, annihilate already inside the source, such that only positrons produced in secondary interactions of charged particles or through primary photons will arrive at a satellite detector. They would have to be produced rather close-by through synchrotron light or bremsstrahlung from ultra-high energy cosmic rays. Pioneering experiments using balloons may indicate an excess of positrons over what is expected by transport models at energies of order $10 \mathrm{GeV}$ [3 5]. Inside the AMS acceptance, one expects several 10 positrons per year with energies above $1 \mathrm{TeV}$.

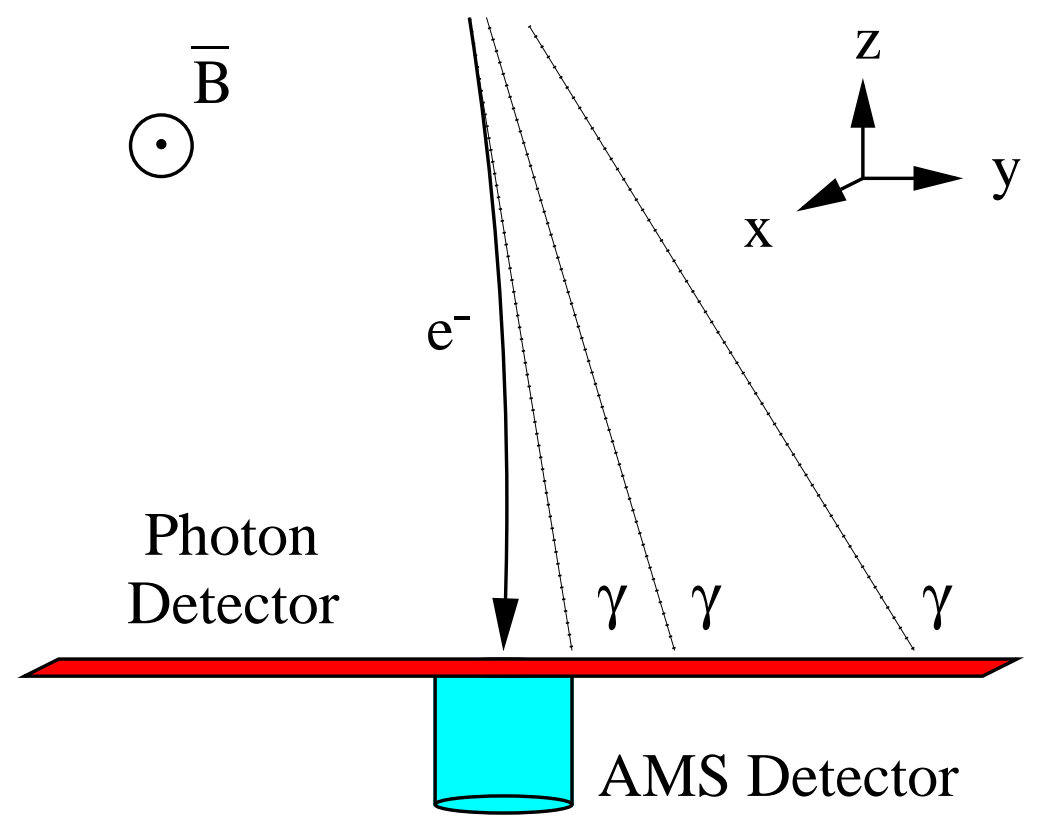

Figure 1: Sketch of the measurement principle of a synchrotron light detector on top of the AMS experiment. For positively charged particles, the synchrotron light photons would be detected to the left of the particles trajectory, for negatively charged particles to the right. The photon spectrometer will be unfolded after installation of AMS on the International Space Station.

For electrons and positrons above about $0.5 \mathrm{TeV}$ impinging on the AMS detector, a charge determination and momentum measurement by curvature in the spectrometer will no longer be possible. Their energy will, however, be measured in the electromagnetic calorimeter. In this paper, we discuss how to obtain an estimate of a particle's momentum and a reliable determination of its charge sign by using synchrotron light emitted in the earth's magnetic field. We propose to install a large surface spectrometer for low energy photons on top of the AMS experiment. Comparison of the energy measurements from the calorimeter to the one derived from the number and energy of synchrotron light photons will allow electron identification.

In addition, ultra high energy nuclei will also produce detectable synchrotron light. As for 
electrons, the position of the synchrotron photons distinguishes particles from antiparticles. Several events per year are expected in the AMS acceptance from energies in the knee region of the primary spectrum.

The principle of this measurement is shown in Fig. 1. Although rather weak, the earth's magnetic field still produces about 20 synchrotron light photons per $100 \mathrm{~km}$ path length, independent of the electron's energy. When some of these photons can be detected in an elongated detector aligned with the magnetic field, photons will be observed on either side of the trajectory depending on the sign of the particle's charge. Moreover, since the synchrotron light energy spectrum depends strongly on the primary particle's energy, the mean energy of the detected photons gives a rough estimate of the primary momentum.

In this paper, we calculate the number of photons expected inside a detector of given length, from an electron with a given momentum transverse to the earth's magnetic field. We calculate the energy spectrum of these photons as a function of the primary momentum. Finally, we extend the discussion to ultra high energy hadrons.

\section{Synchrotron light photons from primary electrons}

In a plane close to the equator, the earth's magnetic field can be approximated by a vector pointing into the direction of the earth's magnetic poles, with magnitude

$$
B(r)=B_{0}\left(\frac{r_{0}}{r}\right)^{3}
$$

with $B_{0} \simeq 0.31210^{-4} \mathrm{~T}$ and $r_{0} \simeq 6.37810^{6} \mathrm{~m}$. In this field, particles with charge $Z e$ follow a helical path with radius of curvature, $\rho$ given by

$$
\rho=\frac{p_{\perp}}{0.3 B Z}
$$

where $p_{\perp}$ is the component of their momentum perpendicular to $\vec{B}$. The mean number of synchrotron light photons, $n$, emitted by a particle with relativistic factor $\gamma=E / m$ on a path of length $\Delta x$ is given by 6 , 8

$$
n=\frac{5 Z^{2} \alpha}{2 \sqrt{3}} \frac{\gamma}{\rho} \Delta x
$$

with the fine structure constant $\alpha \simeq 1 / 137$. Since both the relativistic factor and the radius of curvature are proportional to the particle's energy, this number per unit length is independent of energy for a given particle species.

However, the number of synchrotron photons impinging on a detector of finite size is not constant with energy. If one considers a detector of size $\delta y$ normal to both the particle's momentum vector $\vec{p}=(p, 0,0)$ and the earth's magnetic field $\vec{B}=(0,0, B)$, then photons arrive within $\delta y$ from path lengths roughly equal to $\Delta x \leq L=\sqrt{\rho^{2}-(\rho-\delta y)^{2}}$. The effective path length $L$ then multiplies the rate per unit length from Equ. 3, A more accurate estimation can be obtained when the variation of the magnetic field on the path is taken into account.

The energy spectrum of synchrotron light photons also depends critically on the primary energy. The photon energy spectrum can be expressed as [6]

$$
\frac{d n}{d \epsilon}=n_{0} \int_{\epsilon / \epsilon_{c}}^{\infty} K_{5 / 3}(\xi) d \xi
$$



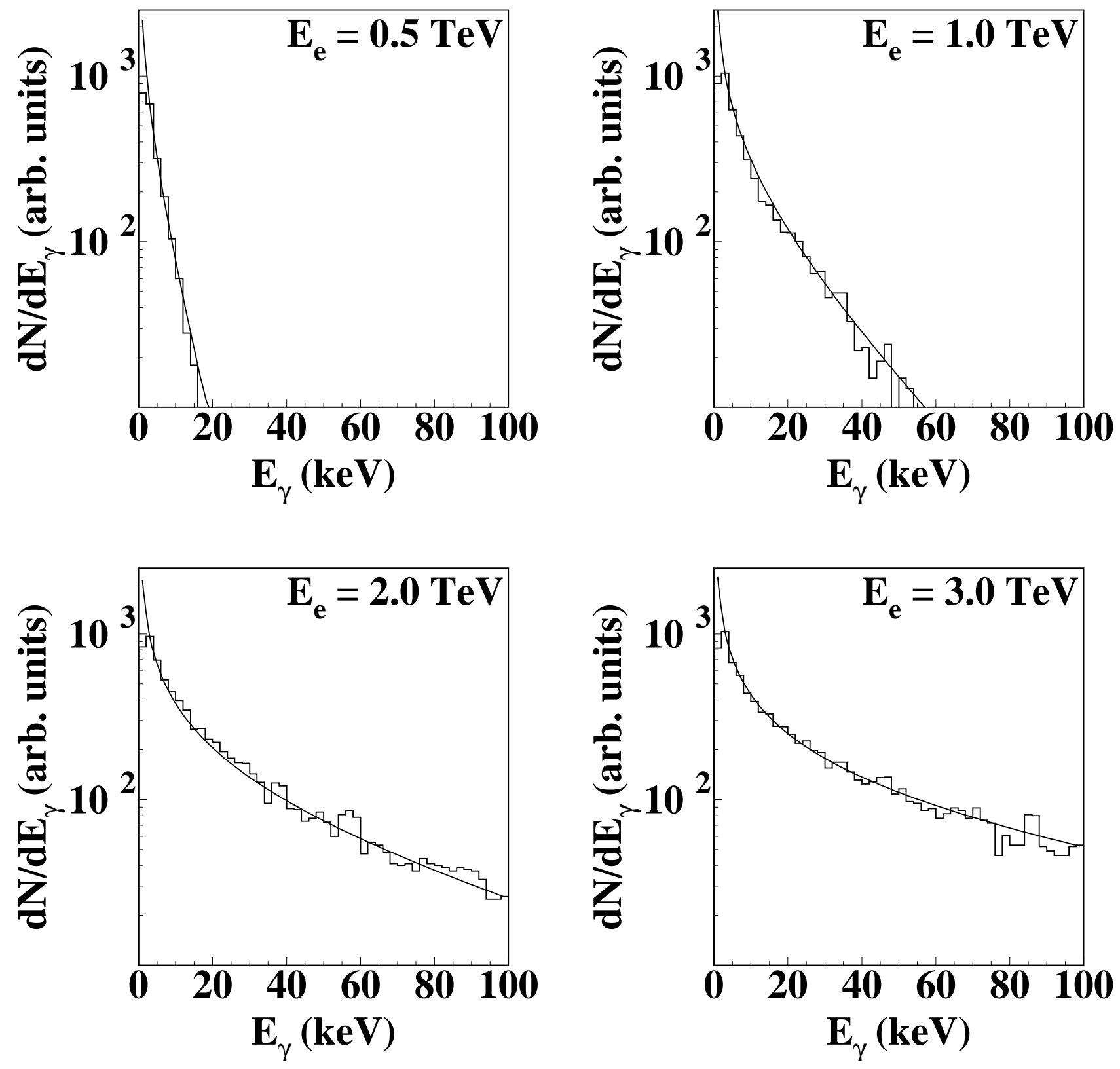

Figure 2: Spectra of synchrotron light photons for different energies of primary electrons. The solid curve is the result of the semi-analytical calculation, the histogram is the simulation result with a low energy cut-off of $5 \mathrm{keV}$. 
with a normalisation constant $n_{0}$, the modified Bessel function $K_{5 / 3}$, the photon energy $\epsilon$ and the characteristic energy $\epsilon_{c}$

$$
\epsilon_{c}=\frac{3}{2} \hbar c \frac{\gamma^{3}}{\rho}
$$

The characteristic energy grows as the square of the primary energy. The fraction of photons, $f$, above a given energy, $\epsilon_{0}$, can be calculated numerically by integrating Equ. 4 and normalising the result. Fig. 2 shows photon spectra at electron energies between $0.5 \mathrm{TeV}$ and $3 \mathrm{TeV}$. The result of the semi-analytical calculation is compared to the simulation of synchrotron light using the GEANT Monte Carlo program [9]. Above a photon energy of $5 \mathrm{keV}$, a cut-off specified in the simulation runs, the spectra agree well.

The mean number of photons, $N_{\gamma}$, inside a detector of width $\delta y$ and above the minimum detectable energy $\epsilon_{0}$, is given by $N_{\gamma}=(n / \Delta x) L(\delta y) f\left(E_{e}, \epsilon_{0}\right)$. The result of the semi-analytical calculation as well as the result obtained from a simulation of electron trajectories using the GEANT package [9] are shown in Fig. 3, as a function of the energy of a primary electron. It is calculated for a very wide detector, $\delta y= \pm 10 \mathrm{~m}$ and a minimum photon energy $\epsilon_{0}=5 \mathrm{keV}$. The results of semi-analytical calculation and simulation agree well. In a wide detector, between 3 and 10 photons above $5 \mathrm{keV}$ would thus on average be detected from primary electron energies between $0.5 \mathrm{TeV}$ and $3 \mathrm{TeV}$. The number of photons observed in a single event will then be sampled from a Poisson distribution with expectation $N_{\gamma}$. The variance of this distribution is indicated in Fig. 3 by the vertical error bars.
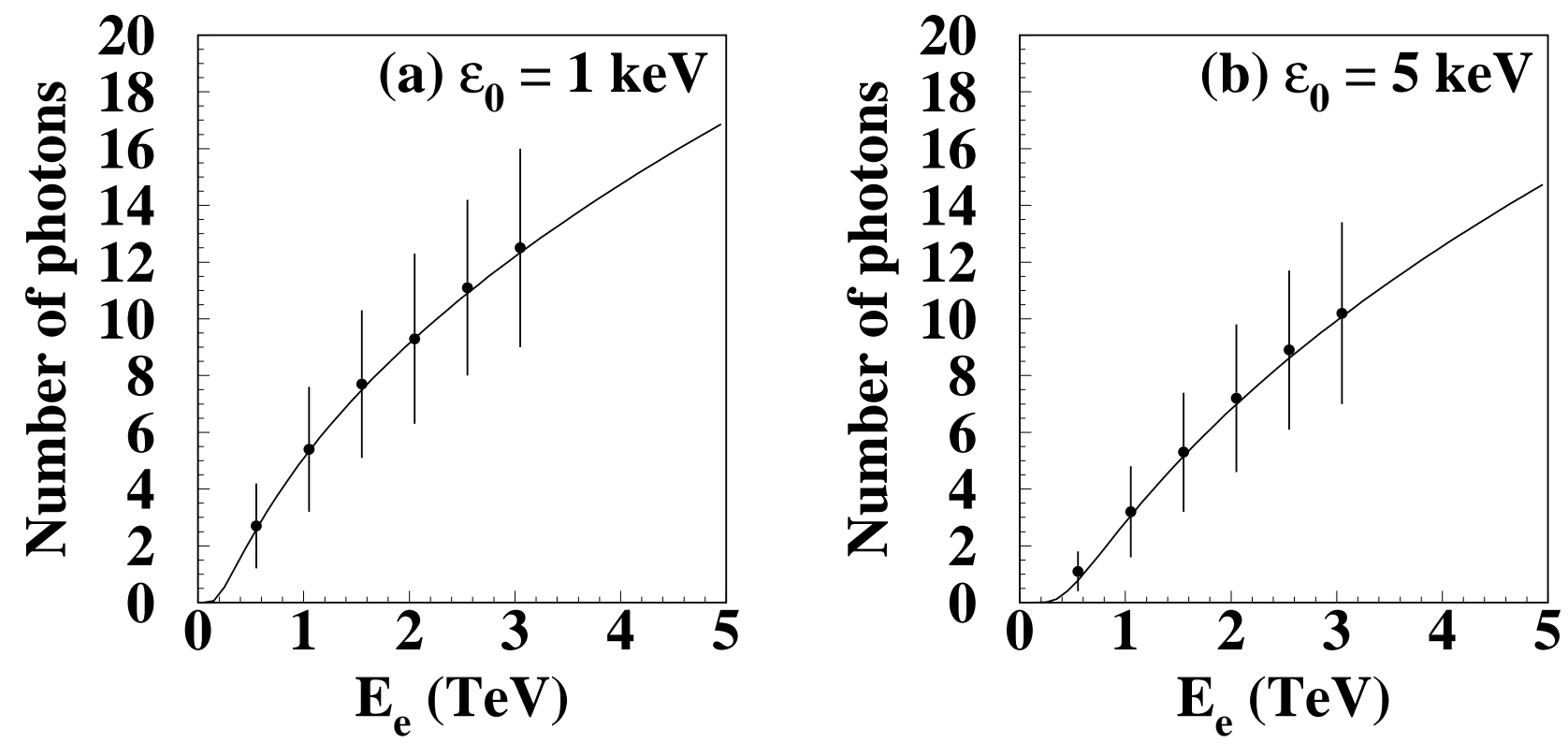

Figure 3: Mean number of photons impinging on a detector of with $\delta y= \pm 10 \mathrm{~m}$ and above a photon energy of (a) $\epsilon_{0}=1 \mathrm{keV}$ and $(\mathrm{b}) \epsilon_{0}=5 \mathrm{keV}$, as a function of the primary electron energy $E_{e}$. The curve is the result of the semi-analytical calculation, the dots indicate the result of a GEANT simulation. The error bars denote the width of the number distribution at each energy.

The fraction of photons inside a smaller detector is shown in Fig. 4 as a function of the detector width $\delta y$. This fraction is almost independent of the primary energy. It is seen that 

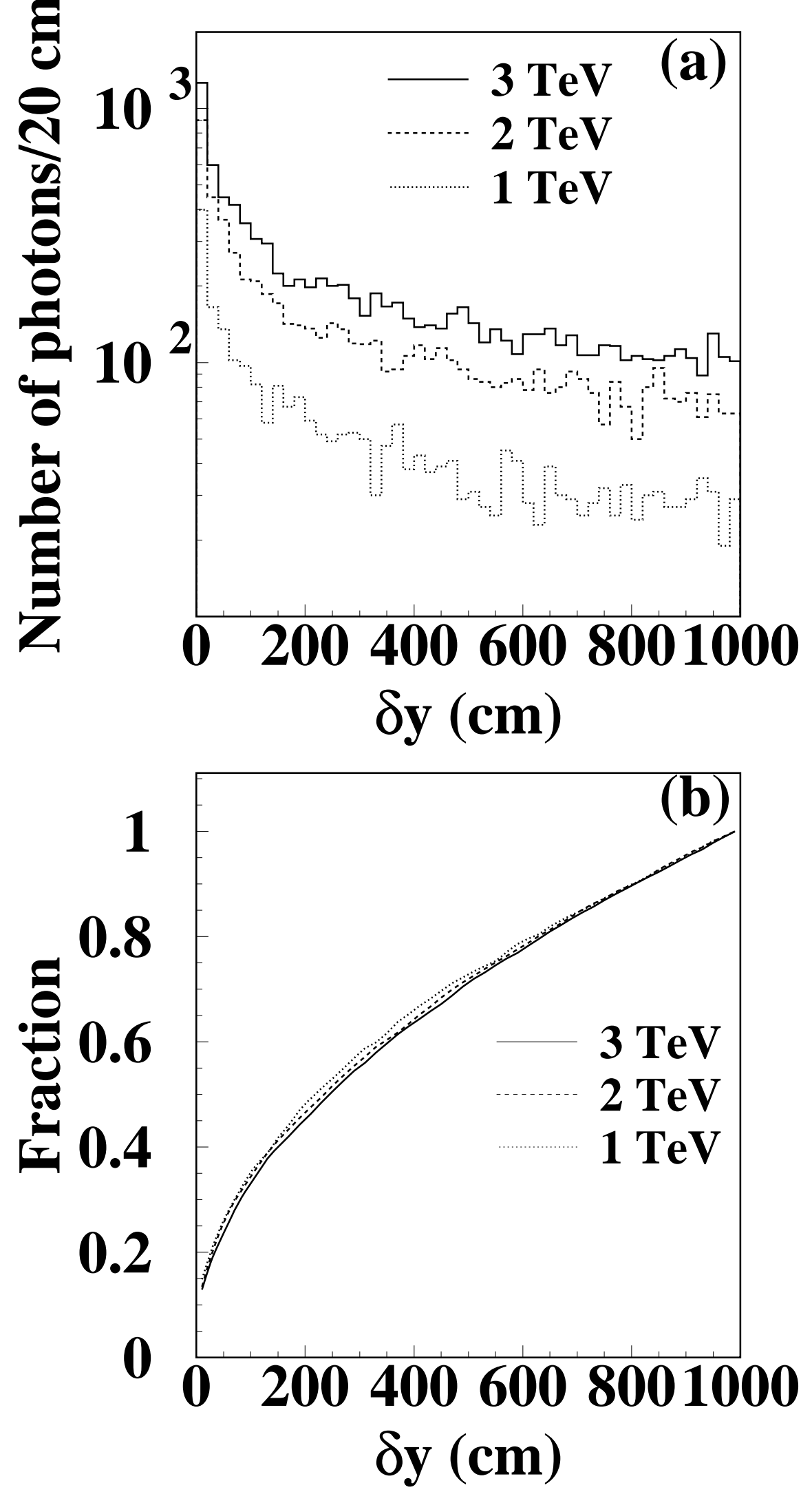

Figure 4: a) Distribution of the distance $\delta y$ between the electron and photons impact on the detector, for 1000 incident electrons at different energies. The shape of the distribution is independent of incident energy and photon energy cut off, while the number of photons is not. b) Fraction of photons impinging on a detector of width $\delta y$, relative to $\delta y= \pm 10 \mathrm{~m}$. This fraction is independent of energy. 
even with a detector as narrow as $\delta y= \pm 2 \mathrm{~m}$, half of the photons read off Fig. 3 would still be visible. The mean number of photons per event would thus be between 1.5 and 5 for a 0.5 $\mathrm{TeV}$ to $3 \mathrm{TeV}$ primary electron in a narrow detector.

Since both the photon energy spectrum and the number of detected photons depends crucially on the primary energy, the number and mean energy of the photons detected in coincidence with the primary electron or positron can be used to get a rough estimate of the primary energy. Fig. 5 shows the mean photon energy and the width of its distribution as a function of the primary energy. The dependence is almost linear. The resolution will be of the order of $30 \%$ from this measurement alone. In addition, a measurement with similar resolution will follow from the relation between the number of photons and the primary energy, as shown in Fig. 3. Combining the two will thus result in an improved measurement, with a total resolution of order 20\%. For electrons and positrons the energy derived from the number and mean energy of synchrotron light photons should be equal to the energy measured in the calorimeter, which has much better resolution. A reliable separation of electrons from nuclei should thus be possible.

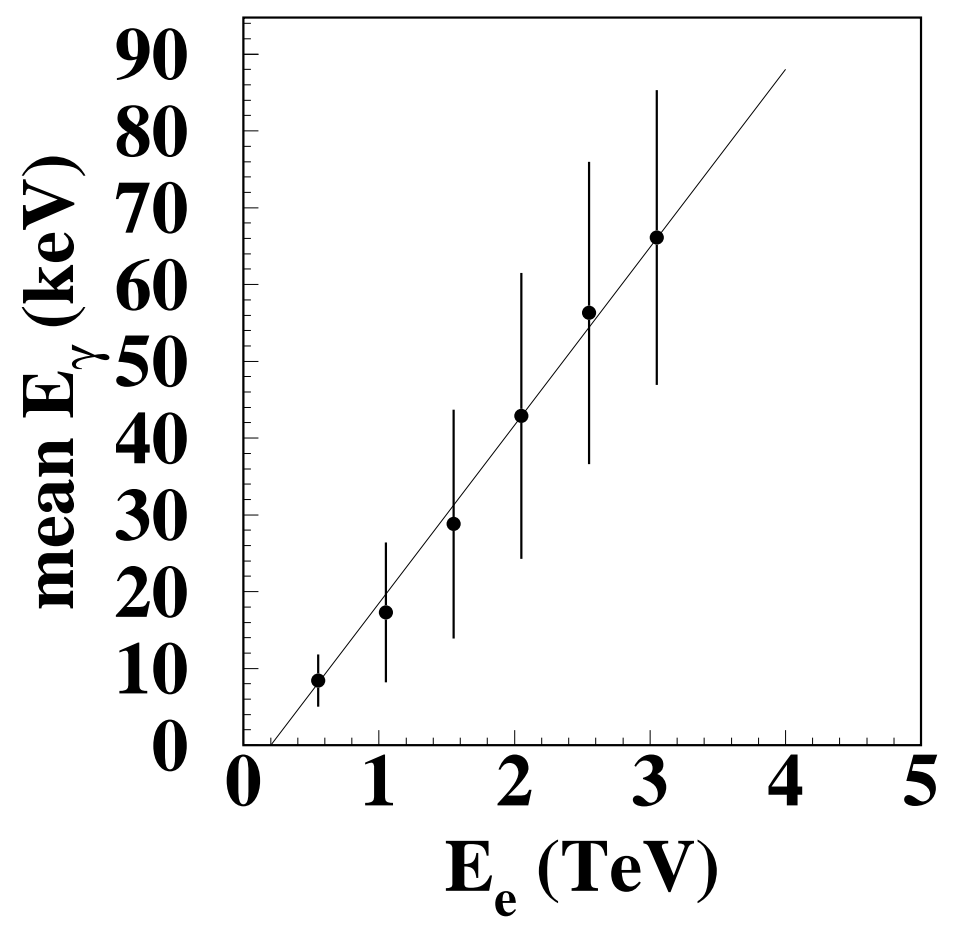

Figure 5: Mean energy of the photons impinging on the detector above a minimum energy of $\epsilon_{0}=5 \mathrm{keV}$, as a function of the primary electron energy $E_{e}$. The relation is basically linear.

\section{Synchrotron radiation from nuclei}

Low energy synchrotron light is also emitted by ultra high energy nuclei. The number of synchrotron photons emitted per unit path length is proportional to $Z^{3} / M$, where $Z$ is the nucleus' charge and $M$ is its mass, and independent of energy. The path length contributing 
to photons impinging on the detector roughly scales like $\sqrt{E / Z}$, where $E$ is the energy of the nucleus, such that energies in the $\mathrm{PeV}$ range give observable photon rates. The photon spectrum is characterised by $\epsilon_{c} \sim E^{2} Z / M^{3}$ and thus in the eV range for the lighter nuclei and PeV energies.

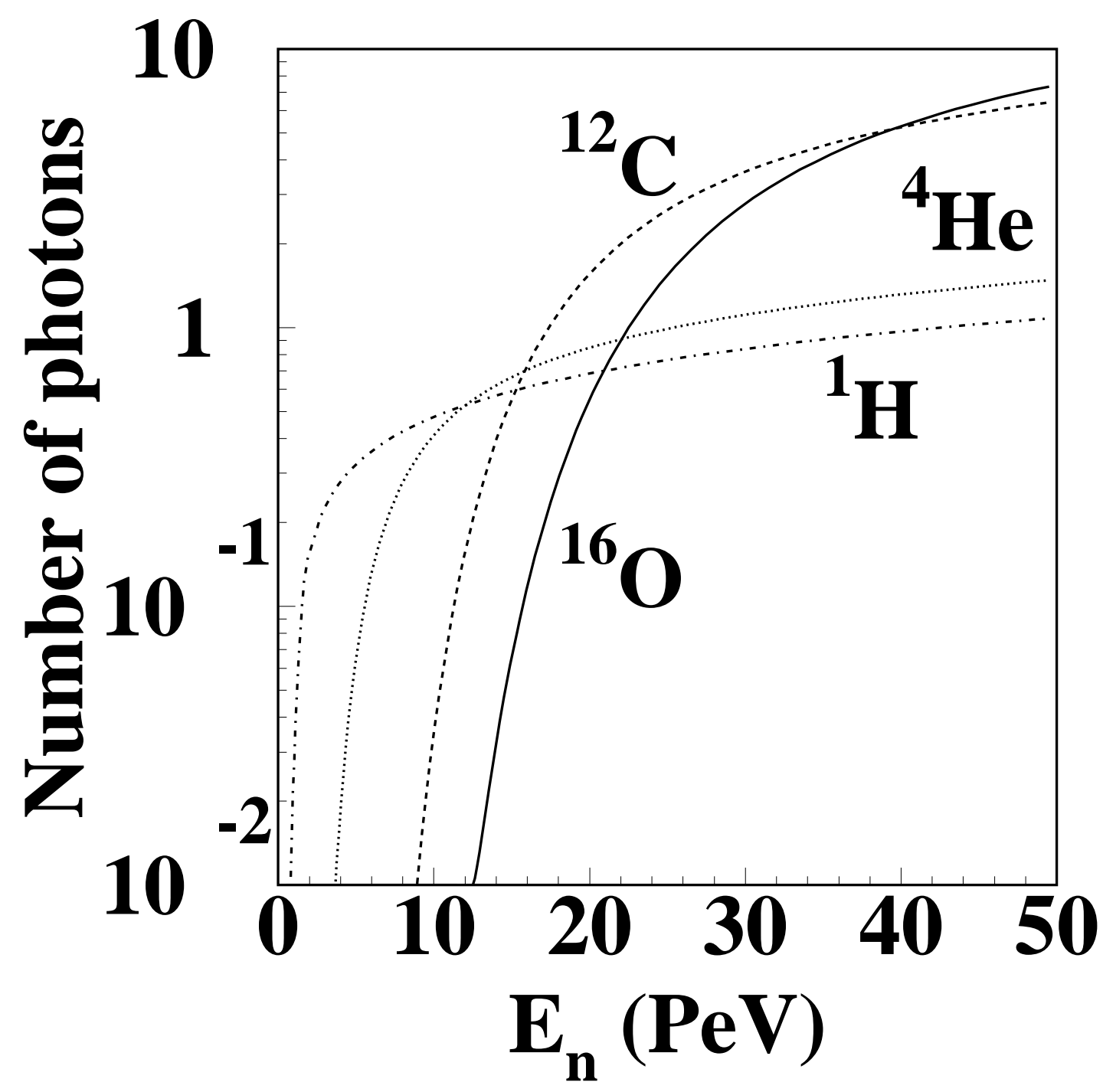

Figure 6: Mean number of photons impinging on a detector of width $\delta y= \pm 10 \mathrm{~m}$ and above a photon energy of $\epsilon_{0}=5 \mathrm{eV}$, as a function of the primary nucleus energy $E_{n}$.

Fig. 6] shows the expected number of photons above $5 \mathrm{eV}$ from protons and light nuclei. The geometrical suppression is not expected to change with respect to what is shown in Fig. 4. Thus, measurable signals are expected for nuclei from the knee region of the energy spectrum. This requires a detector sensitive to photons of a few eV energy. 


\section{Conclusions}

Observable synchrotron light is emitted by TeV electrons and PeV nuclei in the earth's magnetic field. Its position with respect to the primary momentum and the field direction can be used to measure the sign of the particle charge and thus distinguish particles from antiparticles. Moreover, counting the number of synchrotron light photons and measuring their mean energy, an estimate of the primary electron momentum is obtained. This estimate can be used to distinguish electrons from nuclei.

Synchrotron light can be detected and its energy measured using a photon spectrometer of several square meters surface mounted on top of the AMS experiment. It must be sensitive to photon energies above a few $\mathrm{keV}$ for detection of an electron signal, above a few $\mathrm{eV}$ for detection of a signal from nuclei. Candidate technologies for such devices are solid state as well as gaseous and crystal photon detectors.

\section{Acknowledgements}

We wish to express our gratitude to Prof. A. Hofmann, Dr. P. Le Coultre and Prof. A.D. Erlykin for clarifying discussions.

\section{References}

[1] S. Torii et al., $25^{\text {th }}$ ICRC, Durban (1995), Vol. 4, p. 241

[2] S. Ahlen et al., Nucl. Instr. Meth. A 350 (1994) 351

[3] S.W. Barwick et al., Ap. J. 482 (1997) L19

[4] G. Barbiellini et al., A\&A 309 (1996) L15

[5] R.L. Golden et al., Ap. J. 457 (1996) L103

[6] J.D. Jackson, Classical Electrodynamics, John Wiley and Sons, 1962

[7] A. Hofmann, Characteristics of Synchrotron Radiation, CERN Accelerator School 1989, CERN LEP-DI/89-55; A. Hofmann, Theory of Synchrotron Radiation, SSRL ACD-Note 38 (1986)

[8] R. Maier, Synchrotron Radiation, CERN Accelerator School 1990, CERN/91-04

[9] GEANT, Detector Description and Simulation Tool, CERN Program Library Long Writeup W5013 (1994) 